\title{
Operación Cesárea
}

Experiencia personal sobre cincuenta y dos casos

\author{
Dr. Luis Torrado Núñez
}

Del Instituto Materno-Infantil - Bogotá

\section{INTRODUCCION}

La intención del presente trabajo es el comentario, actualización, técnica, indicaciones y resultados materno-infantiles, de la operación cesárea.

\section{MATERIAL Y METODOS}

Se analizan cincuenta y dos (52) intervenciones cesáreas practicadas por el autor en el Instituto Materno e Infantil "Concepción Villaveces de Acosta" de Bogotá, D. E., en las fechas de Marzo $1^{\circ}$ de 1958 a Febrero 28 de 1961.

Las historias clínicas se estudiaron elaborando un cuadro con las siguientes columnas y datos: número de orden, número de historia, iniciales de la paciente, edad de la misma, gestación, paridad, edad del embarazo en semanas, indicación principal de la intervención, indicaciones secundarias, evaluación de la pelvis, exámenes de laboratorio, duración del trabajo de parto, permanencia pre-quirúrgica, condiciones materno-fetales, técnica empleada, duración de la intervención y drogas aplicadas durante ésta. Anestesia: tipo y complicaciones. Niño: peso, tratamiento inmediato, evolución y estado al salir del hospital. Post-operatorio: complicaciones, tratamiento y permanencia post-quirúrgica. Se dejó un amplio margen donde se consignaron principalmente hallazgos quirúrgicos y complicaciones.

\section{COMENTARIO}

\section{Edades}

NN do Casos

De 14 años o menos $\ldots \ldots \ldots \ldots \ldots \ldots \ldots$

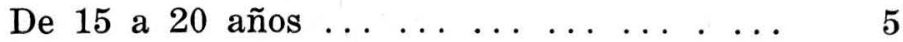




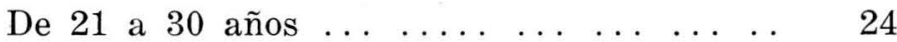

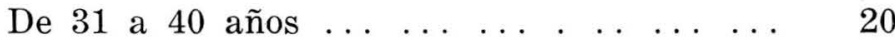

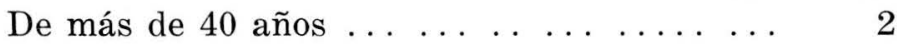

Paridad

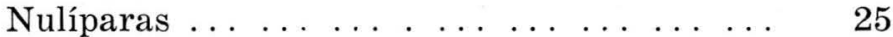

$\begin{array}{lllllllllll}\text { Primíparas } & \ldots & \ldots & \ldots & \ldots & \ldots & \ldots & \ldots & \ldots & & 7\end{array}$

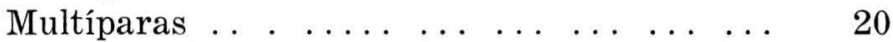

Se registraron seis (6) casos con Cesáreas Previas así:

N de Cesáreas Previas

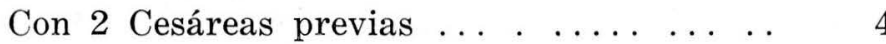

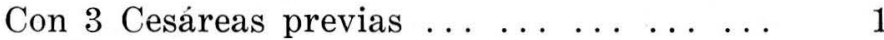

Con 4 Cesáreas previas .................

Edad del embarazo

N de Casos

De menos de 34 semanas . . . . . . . . . . . . . . . 4

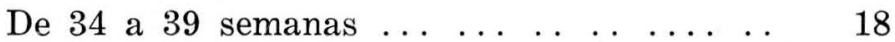

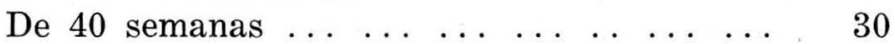

Para mayor claridad hemos agrupado las indicaciones por las cuales se practicaron las cesáreas en: Indicación Principal e Indicación Secundaria.

Los casos más importantes y sus indicaciones serán comentados en detalle más adelante; a continuación haremos un comentario de carácter general por considerarlo de algún interés.

A diferencia de la mayoría de autores que señalan la mayor incidencia de cesáreas con la indicación de "Desproporción céfalo-pélvica" o "Desproporción pelvi-cefálica", en nuestros casos ocupan el primero y segundo lugar las indicaciones: "Situación transversa" y "Sufrimiento fetal" respectivamente. Esto es muy demostrativo de las condiciones de urgencia en que llegan nuestras pacientes al Hospital después de ser "atendidas" en su casa por alguna improvisada comadrona y de haber sido sometidas a torpes maniobras e imprudentes prescripciones farmacológicas. En ocasiones, cuando ingresan, se puede remediar algo, en otras es inútil todo esfuerzo por salvar el hijo o la madre.

Dos de nuestros casos con antecedentes de operación de Man- 


\begin{tabular}{|c|c|c|}
\hline INDICACION PRINCIPAL & No DE CASOS & INDICACION SECUNDARIA \\
\hline Situación transversa & 11 & 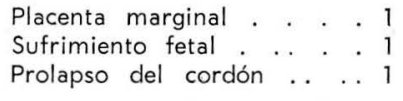 \\
\hline Sufrimiento fetal . . . . . 1 & 9 & 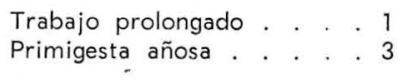 \\
\hline Cesárea previa & 6 & 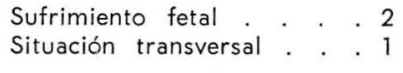 \\
\hline Trabajo prolongado & 4 & 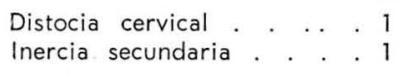 \\
\hline Estrechez pélvica & 4 & 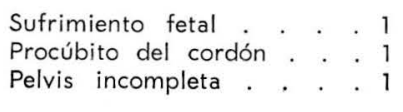 \\
\hline Procidencia del cordón & 4 & Sufrimiento fetal $. \quad \ldots . .2 .2$ \\
\hline Desproporción pelvicefálica & 3 & 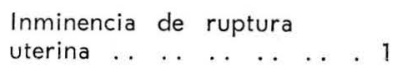 \\
\hline Abruptio placentae & 2 & Sufrimiento fetal . . . . . 2 \\
\hline Distocia ósea & 2 & \\
\hline Falta de encajamiento & 1 & \\
\hline Inminencia de ruptura uterina & 1 & \\
\hline Distocia cervical & 1 & \\
\hline Distocia de partes blandas & 1 & \\
\hline Presentación de frente & 1 & \\
\hline Tetanización uterina & 1 & \\
\hline Placenta previa total & 1 & \\
\hline
\end{tabular}


chester determinaron operación cesárea, son los que figuran con las indicaciones de "Distocia Cervical" y "Distocia de partes blandas". Esta última tenía como secuelas de la intervención ginecológica, bridas vaginales.

\section{EVALUACION DE LA PELVIS}

En términos generales nuestra conducta al respecto fue, en los casos que la permitían, la prueba de trabajo. No nos ceñimos a ningún plan preestablecido, sino que tuvimos en cuenta otras condiciones tales como la paridad, dinámica uterina, condiciones maternas y principalmente fetales. Aparecen 13 de nuestras pacientes con valoraciones relativas como lo es la pelvimetría externa. En 20 pacientes se registran evaluaciones más o menos precisas como los datos obtenidos por el tacto vaginal. En el resto de los casos consideramos innecesario e imprudente intentar alguna valoración por las características mismas de algunas distocias: situacion transversa, procidencia del cordón, placenta previa, abruptio placentae, sufrimiento fetal manifiesto, algunas cesáreas previas, etc.

Los dos casos señalados como distocias óseas fueron debidos, uno a pelviviciación por artropatía infecciosa bilateral de la cadera adquirida en la infancia en paciente GIII Cesáreas: II y antecedentes de pre-eclampsia en el segundo embarazo. El otro, se trataba de una GIII PI Cesárea: I con parto espontáneo (segundo embarazo) de feto muerto y quien además padecía de una malformación congénita del miembro inferior izquierdo con acortamiento real y aparente y marcada atrofia muscular, esta pelvis la consideramos, etiológicamente una pelvis oblicua.

\section{EXAMENES DEL LABORATORIO}

En 28 casos no se practicó ningún examen de laboratorio. En 15 análisis más o menos completos como: C.H., clasificación, azoemia, glicemia, coprológico, parcial de orina, fotofluorografía, serología. En 9 pacientes se obtuvo únicamente el dato de la proteinuria. Es importante aclarar que la mayoría de las pacientes nuestras llegan al Instituto únicamente para la atención de su caso y que son pocas las que asisten al control prenatal. Posteriormente a la reorganización del servicio de Toxemias se practica de rutina la prueba del ácido sulfosalicílico. 


\section{DURACION DEL TRABAJO}

Tan solo consideraremos los casos intervenidos con diagnóstico de "Trabajo Prolongado" y como tal entendemos al mayor de veinte horas.

De los 4 casos operados con indicación principal de trabajo prolongado, dos fueron debidos a una errada determinación de la hora de iniciación del trabajo por datos mal suministrados por la paciente. Los 2 restantes, uno por distocia cervical (dilatación estacionaria) y el otro por inercia uterina secundaria la cual se trató inicialmente por medios farmacológicos, sin resultados.

\section{PERMANENCIA PRE QUIRURGICA}

A pesar de los innumerables inconvenientes que en nuestra práctica se presentan, es necesario anotar cómo algunos casos fueron resueltos de acuerdo a las características de urgencias de éstos.

\section{CONDICIONES MATERNAS EN EL MOMENTO DE LA INTERVENCION}

En nuestras pacientes figuran 7 casos de Preeclampsia y 2 de Eclampsia sin ser éstas las indicaciones que indujeran a la operación ya que entre nosotros prima el concepto de que el tratamiento de esta entidad es eminentemente médico. Se distribuyeron así:

PREECLAMPSIA

ECLAMPSIA

$$
N^{\circ} \text { de Casos }
$$

7

2

\section{Indicación}

$\begin{array}{lll}\text { Suf. Fetal . . . . } & 3 \\ \text { Dist. Osea . . } & 1 \\ \text { Tetanización } & & \\ \text { uterina . . . . } & 1 \\ \text { Desproporción } & & \\ \text { Pelvicefálica . . } & 1 \\ \text { Estrechez } & & \\ \text { Pélvica . . . . } & 1 \\ \text { Suf. Fetal . . . . } & 2\end{array}$

Los dos casos últimos se trataban de primigestantes de 40 años - registramos además dos casos de anemia aguda y 2 de infecciones respiratorias-. El resto de nuestras pacientes lo consideramos como de aceptables o buenas condiciones generales. 


\section{CONDICIONES FETALES}

Calificamos 27 casos como en malas condiciones, los mismos intervenidos con indicaciones principal o secundaria de "sufrimiento fetal". Dos casos con infección amniótica marcada y prematuridad.

\section{TECNICA QUIRURGICA}

Practicamos generalmente la técnica conocida como cesárea segmentaria transversal transperitoneal. Con alguna frecuencia empleamos para la extracción fetal, cuando ésta la presumimos laboriosa, una o ambas ramas del fórceps pequeño de Simpson con lo cual tratamos de evitar desgarros uterinos. De rutina hacemos extracción manual de la placenta seguida de cuidadosa limpieza de la cavidad uterina y aplicamos 10 unidades endovenosas de Pitocín. Respecto a la reconstrucción de la pared, excepción hecha de los casos con laparotomías previas, siempre hacemos el afrontamiento aponeurótico con catgut cromado y sourcet simple con lo cual disminuímos considerablemente el tiempo quirúrgico. No se han presentado casos con dehiscencias parciales o totales de suturas a pesar de que algunos hicieron infección de la cicatriz. En 8 de nuestras pacientes intervenidas posteriormente por otros cirujanos no se hace ningún reparo respecto a la confrontación tisular.

En un caso que encontramos la placenta insertada a la altura de la cara anterior del segmento, hicimos extracción previa de ésta, maniobra que si se realiza con rapidez es mucho menos agresiva tanto para la madre como para el feto, que si se trata de incidirla para hacer la extracción, con lo cual no hacemos sino aumentar la hemorragia y por lo mismo la anoxia fetal que tratamos de evitar y que se acentúa con el inevitable desprendimiento de la placenta que se intenta franquear.

\section{DROGAS APLICADAS DURANTE LA INTERVENCION}

Aparte de ciertas indicaciones hechas por nosotros como transfusiones, fenobarbital y sulfato de magnesia en pacientes toxémicas, la mayoría de las drogas empleadas son ordenadas por el Departamento de Anestesia. De rutina se usa dextrosa al 5\% en agua destilada; se emplearon además otros fármacos como: Anectine, Diparcol, Morfina, Efedrina, Atropina, Cedilanid, y 
Fenergán. Por razones de tipo netamente farmacológico que adelante expondremos, es necesario anotar que drogas como el Anectine, Diparcol y Morfina no deben ser indicados antes de la extracción fetal, los restantes se pueden emplear en cualquier momento de la intervención.

ANECTINE. RUBILEXIN: (cloruro de succinil colina). Su acción se ejerce sobre la musculatura estriada. Aunque se dice de efecto "curarizante" su farmacodinamia es diferente ya que el curare actúa sobre la placa neural del músculo "desconectándola" y en cambio éste parece que repolariza la acción neural podríamos decir "tetanizándola" por lo que paraliza su acción. Se emplea como relajador de la musculatura abdominal y para intubación.

MORFINA. - El más potente analgésico conocido de efecto narcótico es asímismo el fármaco de más cuidado por sus efectos tóxicos colaterales que se manifiestan inicialmente por euforia, pulso rápido, luego vértigo, náuseas, lipotimia, pulso lento y débil, miosis, respiración lenta y cianosis; posteriormente: respiración de Cheyne-Stokes, coma y muerte. Debe evitarse su uso por la profunda depresión del centro respiratorio materno y fetal.

DIPARCOL. - (dietiletildibenzoparatiazina). Recomendado como excitante del centro respiratorio y sedante del S.N.C. (??) en la práctica produce excitación sicomotriz. Su empleo es cada vez menor.

FENERGAN. - (dimetilmetiletildibenzoparatiazina). Antihistamínico y sedante del S.N.C., usado generalmente como medicación preanestésica. En nuestros casos se empleó para controlar reacciones de tipo alérgico.

CEDILANID. - (lanatósido C) Utilizado para estimular la acción vagal, especialmente para reforzar la potencia de la contracción cardíaca aunque disminuye el ritmo de ésta. Indicado por vía venosa es el digitálico de más rápida acción, su máximo efecto se obtiene a los 40 minutos.

ATROPINA. - (atropa belladonna). Quizá la droga más socorrida. Posee marcada acción parasimpaticolítica, relaja la fibra muscular lisa, dilata la pupila, aumenta el ritmo cardíaco y respiratorio (estímulo sobre la corteza cerebral). Localmente es un anodino. Se emplea especialmente para inhibir las secreciones. 
EFEDRINA - Simpaticomimético que actúa especialmente sobre las terminaciones nerviosas de los vasos sobre los cuales ejerce acción presora. Se emplea, por lo mismo, para controlar la acción hipotensora que produce la raquianestesia.

\section{RESULTADOS MATERNOS \\ COMPLICACIONES DURANTE LA INTERVENCION}

Tuvimos 5 casos de desgarro segmentario con compromiso de la arteria uterina izquierda en dos de ellos. Se distribuyen así:

$1^{\circ}-\mathrm{N}^{\circ}$ de orden $9-$ Paciente: M. M. GIII PI, intervenida con indicación de "Distocia de partes blandas" por bridas vaginales debidas a intervención tipo Manchester practicada probablemente después del primer embarazo. Permanencia prequirúrgica: 9 horas Duración de la intervención: 1:30 horas. Se aplicó plasma durante ésta. Niño clasificado al nacer: bueno. Peso: 2.900 grs. Presentó infección de la herida en el postoperatorio y tuvo una permanencia de 20 días.

$2^{\circ}-\mathrm{N}^{\circ}$ de orden: 14. - Paciente: E. M. GIII PII CI. Primer parto con cesárea, segundo por vías naturales. Duración del trabajo: 11:30 horas. Duración de la intervención: 1:05 horas. Indicación: "Inminencia de ruptura uterina". Niño con Apgar de 10/10 y peso de 3.400 grs. Hizo infección de la cicatriz y tuvo una permanencia de 12 días.

$3^{\text {o }}$ - No de orden: 15. - Paciente F. D. GIII PII. Indicación: "Situación transversa". Permanencia prequirúrgica: 1:10 horas. Duración de la intervención: 1:10 horas: Se administró Peristón. Niño con Apgar 10/10 y peso de 2.580 grs. No hizo ninguna complicación postoperatoria y permaneció 6 días en el hospital.

$4^{\circ}-\mathrm{N}^{\circ}$ de orden: 20. - Paciente C. P. P. GXIII PXII. Indicación: “'Sufrimiento fetal". Parto anterior intervenido por "Presentación de frente". Duración del trabajo: 1:25 horas. Duración de la intervención: 40 minutos. Se hizo intento de fórceps antes de la operación. Se aplicó plasma. Niño: 10/10 y peso de 3.700 grs. No presentó ninguna complicación ulterior y tuvo una permanencia de 8 días.

$5^{\circ}$ - No de orden: 36. - Pcciente: A. R. G. - GV. PIV. Indicación: "Situación transversa". Duración del trabajo: 3:05 horas. Duración de la intervención: 40 minutos. Niño: $10 / 10$ y peso de 3.100 grs. Se aplicó sangre total posteriormente. No hizo complicaciones en el postoperatorio y salió a los 8 días.

Podemos concluír que la complicación más frecuente durante la práctica de la Cesárea es el desgarro muscular del útero con compromiso en ocasiones de algún vaso de importancia, accidente éste que puede ser fatal si no se descubre a tiempo, de ahí que se aconseje el rápido reparo de la herida uterina poniendo especial cuidado en sus ángulos. Este tipo de accidentes se presenta 
con mayor frecuencia en aquellos casos en que se produce una sobredistensión del segmento y cuando se aumenta éste al practicar maniobras intracavitarias. El mismo riesgo está aumentado en úteros con cicatrices anteriores. Por esto creemos que toda precaución que se tome al respecto es poca y nos declaramos conformes a las normas de nuestro Instituto de no permitir este tipo de intervenciones sino a personal suficientemente adiestrado.

\section{MORBILIDAD MATERNA}

Las complicaciones postoperatorias se distribuyen así:

Tipo de Complicación No de Casos

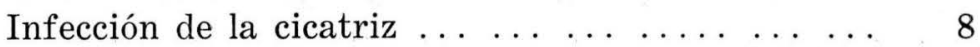

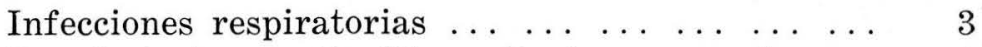

Ileo dinámico, peritonitis y sicosis puerperal . . . . 1

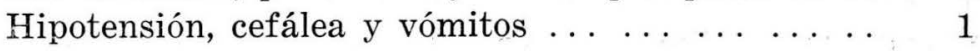

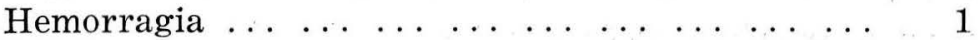

Creemos que esta incidencia de infecciones sea debida a una falta de servicios especiales para operadas y a falta de preparación del personal auxiliar, siempre insuficiente, que debe atender a pacientes de variada patología.

\section{TRATAMIENTO}

De rutina indicamos la movilización temprana. No descubrimos la herida ni ordenamos cambio de apósito sino hasta el tercer día. Prescribimos: líquidos, antibióticos, ácido ascórbico, calmantes (casi nunca opiáceos), estimulantes de la retracción uterina $\mathrm{y}$ del peristaltismo intestinal.

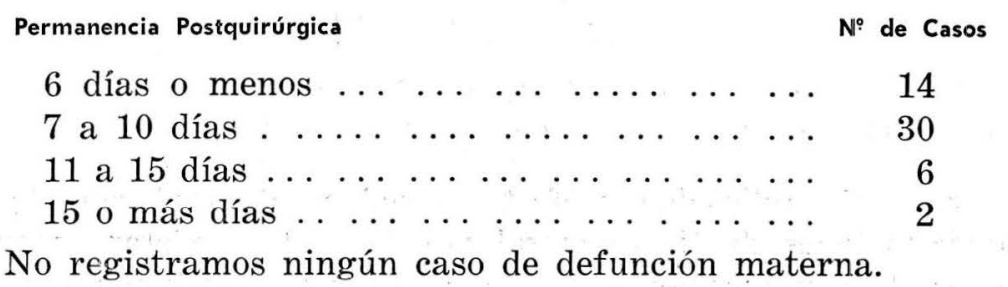

ANESTESIA EMPLEADA

Tipo de Anestesia

$N^{\circ}$ de Casos

Raquídea ....................... . . . 50 
Raquídea y Pentothal . ... . . . . . . . . . . 1

Raquídea, ciclo y éter . . . . . . . . . . . . . . . $\quad 1$

El caso en el cual se empleó el Tiopental sódico fue el de una paciente intervenida con indicación de "Distocia Osea" por artropatía infecciosa bilateral de la cadera y en la cual no se sospechó su estado toxémico e hizo dos convulsiones durante el acto quirúrgico y este fármaco fue indicado como sedación anticonvulsivante más que como anestésico. En el otro caso en que se administró éter y ciclopropano, no surtió efecto la raquianestesia.

De lo anterior podemos concluír que la anestesia raquídea fué utilizada en todos los casos con lo cual estamos de acuerdo con otros autores que es ésta hoy día la anestesia de elección para la operación cesárea, sin intentar afirmar que ésta sea ideal pues si bien es cierto que en el mayor número de nuestras pacientes (44) no se nos presentó ningún tipo de complicaciones en las 8 restantes pudimos comprobar algunas caracterizadas por hipotensión arterial, calofrío, cefálea, excitación y epigastralgia. Concluiremos diciendo que la raquianestesia ofrece un mayor número de ventajas para la madre y para el feto pero que también presenta algunos inconvenientes los cuales analizaremos a continuación.

\section{VENTAJAS PARA LA MADRE}

$1^{\text {a }}$ - No se emplea medicación preanestésica.

En la amplia experiencia del departamento de anestesiología, se ha llegado a la conclusión de que la cesárea es una intervención de urgencia y que como tal no se puede esperar el efecto de la medicación prequirúrgica, con esto se ha logrado y sin ningún riesgo evitar la acción siempre indeseada de estos fármacos.

$2^{a}$ - Produce una perfecta indolorización y la más efectiva acción relajante de la musculatura abdominal.

$3^{a}-\mathrm{Su}$ aplicación y efecto se reduce al canal raquídeo sin manifestaciones de tipo general por lo tanto existen menores riesgos de complicaciones en pacientes con cardiopatías compensadas, enfermedades renales y respiratorias. Vale anotar los casos muy frecuentes de nuestras pacientes a las cuales antes de su llegada al hospital se les ha intentado "estimular" su trabajo de parto con una sobrecarga alimenticia que viene a complicar el 
pronóstico por el inevitable reflejo del vómito y la consiguiente aspiración del contenido gástrico, fenómeno éste más frecuente en la anestesia de tipo general.

$4^{\text {a }}$ - Por último, como partidarios que somos de la preparación sicoprofiláctica de la embarazada para el acto más sicosomático de la naturaleza creemos que la operación cesárea a diferencia de otras intervenciones de las cuales afirman algunos que constituyen un verdadero trauma síquico del cual no se debe tener o evitar la vivencia, en la madre ya frustrada por su distocia, se debe dejar conciencia al menos de que "pare por el vientre". Nunca hemos escuchado una protesta, un desistimiento de la mujer abocada a la solución quirúrgica de su parto. Aun más, aquella desgraciada que rechaza la maternidad, ante el peligro que amenaza su fruto parece cambiar su postura inicial y que sentimientos redentores la asistieran a última hora. Esto parece indicar que existe un acondicionamiento saludable y positivo de la mujer ante la maternidad sea cual fuere la condición como ésta se presente. Muy distinta es la situación de rechazo o desconfianza de cualesquier otro paciente frente a otra clase de intervención.

\section{VENTAJAS FETALES}

$1^{\text {a }}$ - Si afirmamos que no es necesaria la medicación preanestésica, tenemos que aceptar igualmente que evitamos el empleo de drogas que afectarían el metabolismo fetal.

$2^{\text {a }}$ - El anestésico empleado, por las mismas razones expuestas anteriormente, no pasa la barrera placentaria como lo hacen los empleados en anestesia general.

$3^{a}$ - Las maniobras de reanimación son mucho más fáciles.

\section{DESVENTAJAS}

Más propiamente que desventajas podríamos llamarlas contraindicaciones que se pueden resumir así: la raquianestesia si no es controlada con agentes vasopresores dada su acción vagal tiende a producir hipotensión, de ahí, que no se deba emplear en los estados hemorrágicos, en las anemias severas o en aquellas entidades hipotensivas como la insuficiencia suprarrenal. Ahora bien, como parece estar demostrado que una presión sistólica me- 
nor de 6 es insuficiente para mantener el riego placentario, es indudable el riesgo que se corre si no se hace un cuidadoso empleo de esta clase de anestesia que podría favorecer un estado de anoxia fetal.

\section{RESULTADOS FETALES}

Por norma general en nuestro Hospital la atención inmediata del recién nacido en toda distocia, es encomendada al pediatra. De rutina se le practica aspiración nasal, oral y gástrica, según el caso, maniobras de reanimación o terapia profiláctica.

No pudimos hacer una clasificación única del recién nacido debido a que en la primera parte de nuestras historias figura la antigua valoración de bueno, regular o malo. La clasificación de Apgar ha sido adoptada en los últimos años.

Se distribuye como sigue:

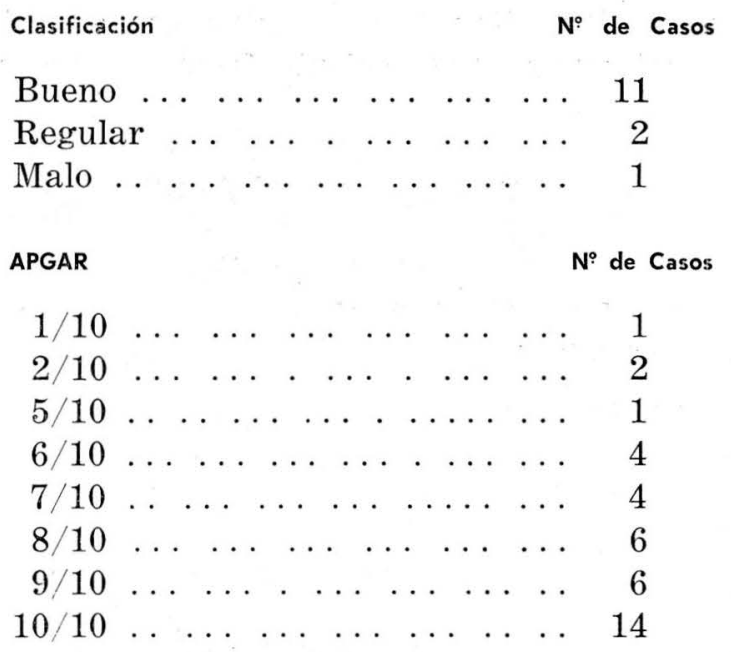

No registramos ningún caso de muerte perinatal.

Morbilidad Infantil

№ de Casos

Infecciones respiratorias . . . . . 5

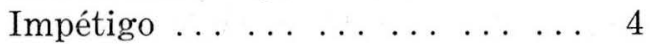

Conjuntivitis ............ $\ldots 3$

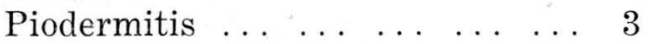




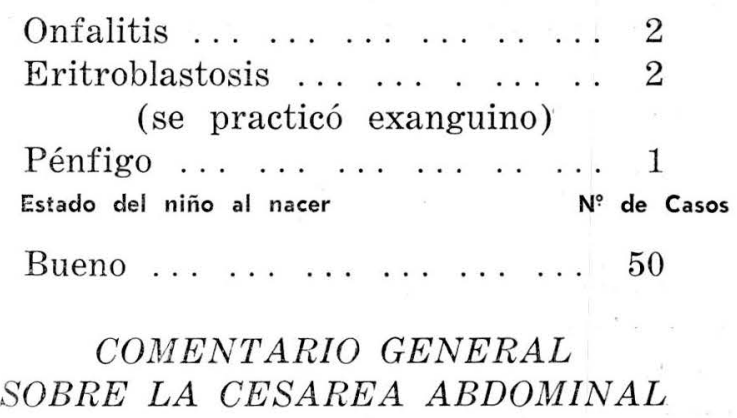

Consideramos la Cesárea como intervención de urgencia sobre todo en su primera fase o sea hasta la extracción fetal, por lo tanto siempre nos procuramos un buen campo operatorio practicando una incisión suficiente. Seguimos creyendo en el viejo aforismo de "Incisiones pequeñas, complicaciones grandes".

Respecto a otras técnicas como la descrita originalmente por Opitz (segmentaria longitudinal) y defendida por algunos, la creemos de alguna utilidad ya que la hemos precticado posteriormente a este trabajo, en los casos que está formalmente indicada pero que el peligro del desgarro se presenta también con esta técnica sobre todo en el cuello uterino.

Finaimente creemos que la cesárea ha evolucionado como técnica quirúrgica pero que puede ser perfeccionada, sobre todo ahora cuando la introducción de técnicas anestésicas, transfusiones y antibióticos la hacen cada vez más libre de riesgos lo cual ha contribuído a prodigarla más como solución a muchos problemas obstétricos. Sinembargo esto no debe inducirnos a que este progreso deba supeditar la conservación del espíritu crítico y el empleo juicioso de los diversos recursos tocológicos.

\section{CUADROS COMPARTIVOS}

Incidencia de partos, cesáreas y causas de mortalidad postquirúrgica en el Instituto Materno-Infantil, durante la misma época de nuestras experiencias:

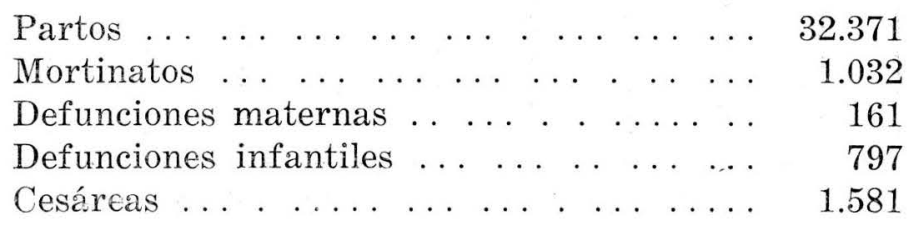


Esto nos da una incidencia de la operación Cesárea del $4.8 \%$.

Causas de mor:alidad por Cesárea

N de Casos

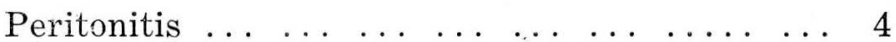

( 1 caso de cesárea vaginal)

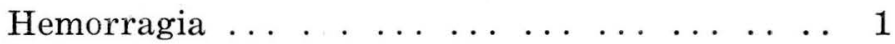

$\begin{array}{llllllllll}\text { Paro cardíaco } & \ldots & \ldots & \ldots & \ldots & \ldots & \ldots & \ldots & \ldots & 1\end{array}$

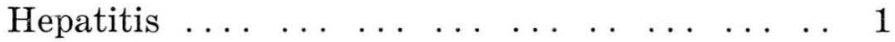

$\begin{array}{lllllllllllll}\text { Embolia } & \ldots & \ldots & \ldots & \ldots & \ldots & \ldots & \ldots & \ldots & \ldots & \ldots & 1\end{array}$

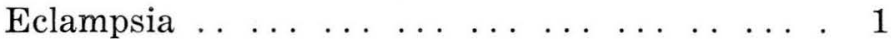

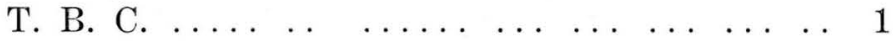

Las anteriores causas de muerte, sin depurar, nos darían un porcentaje del $1.5 \%$.

PIERRE VELLAY, sobre 1.000 pacientes preparadas con el método sicoprofiláctico comunica únicamente 12 intervenciones cesáreas y junto con LAMAZE afirman que esta preparación sirve como premedicación al acto quirúrgico, comprobando en casos de pacientes preparadas:

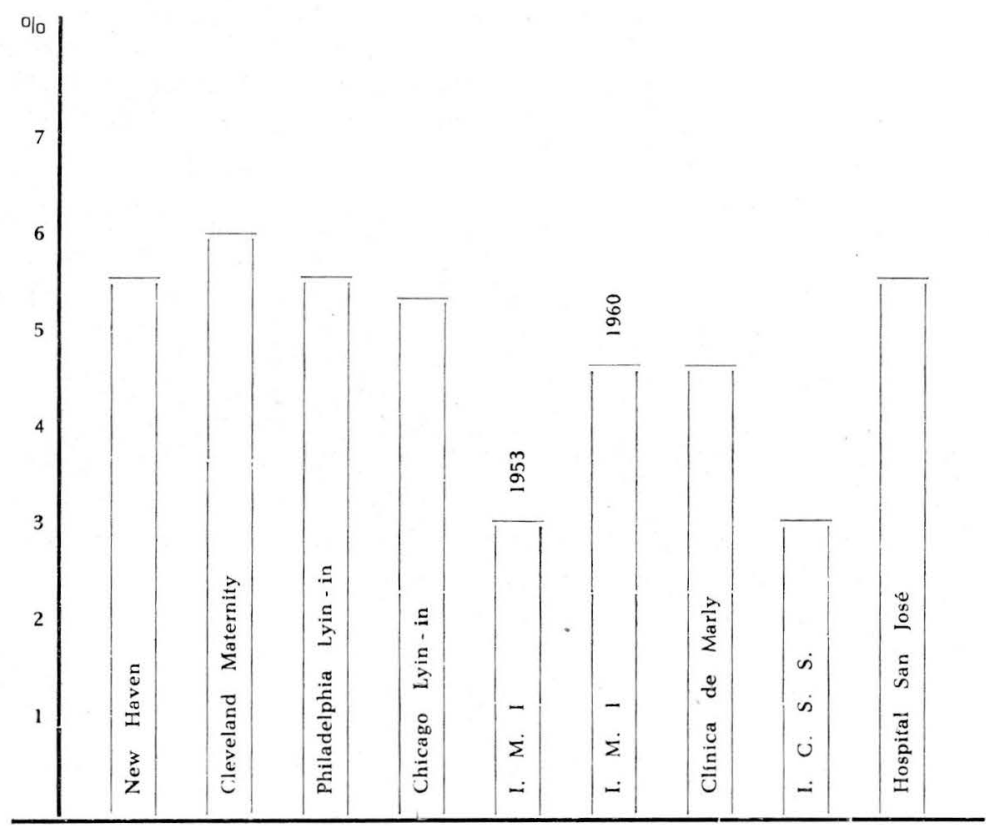

Incidencia de la operación cesárea en diferentes instituciones 
- un adormecimiento mucho más rápido que en las mujeres no preparadas con una dosis anestésica reducida a más de la mitad.

- la duración del acto operatorio es netamente reducida.

- los riesgos anestésicos se disminuyen para el niño.

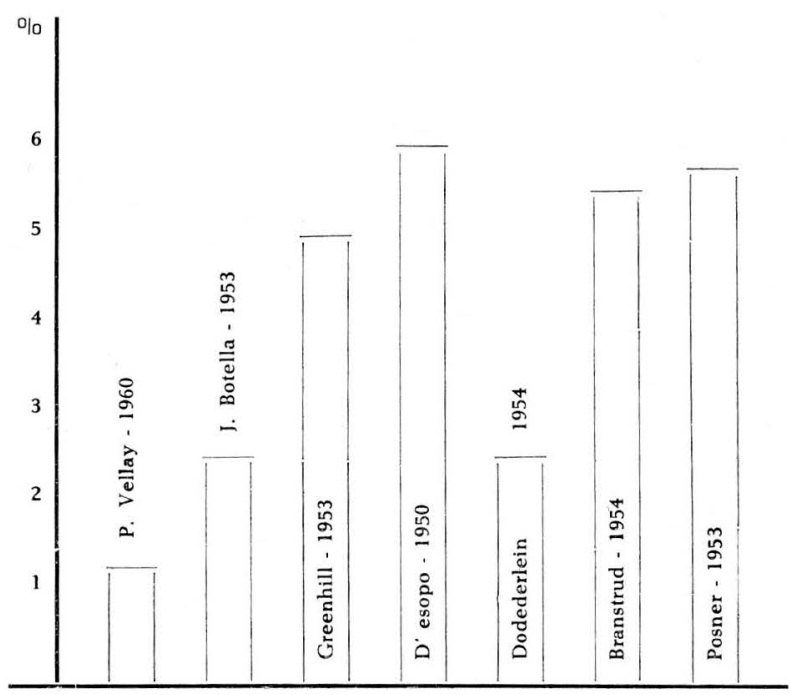

Incidencia de la cesárea según diferentes autores

\section{BIBLIOGRAFIA}

BOTELLA LLUSIA: "Tocurgia”. Ed. Científico Médica. Barcelona. 1957.

MARTINEZ SAENZ CARLOS: "Operación Cesárea en la Clínica $1^{\mathbf{0}}$ de Mayo". Meinorias de la Primera Convención de Obstetricia y Ginecología de 1953. Revista de la Sociedad Colombiana de Obstetricia y Ginecología. Mayo-Junio de 1954.

MORAGUES BERNAT: "Obstetricia”. 1957.

LAMAZE y VELLAY: "Psychoprofhylaxie et travail dirige". Journees Franco-Suisse de Obstetrique et Ginecologie. Bale Juillet 31957.

MUÑOZ DELGADO SAULO, COBO COBO EDGAR: "Indicaciones selectivas de la Cesárea segmentaria longitudinal". Separata de la Revista de la Sociedad Colombiana de Obstetricia y Ginecología.

FALCONER and PATERSON: "Current Drug Handbook". W. B. Saunders Company. 1960.

LEON JUAN: “Obstetricia”. Editorial Científico Médica. Buenos Aires. 1957. VELLAY PIERRE: "Parto dirigido en pacientes preparadas". Boletín de la Sociedad Internacional de Sicoprofilaxia Obstétrica. Marzo 1960. 